\title{
Phasic and Tonic Cells in the Cat's Lateral Geniculate Nucleus
}

\author{
Yoshiro Fukada and Hidfaki Saito \\ Research Group on Auditory and Visual Information Processing, \\ NHK Broadcasting Science Research Laboratories, Kinuta, \\ Setagaya-ku, Tokyo
}

Fukada, Y. and SaIto, H. Phasic and Tonic Cells in the Cat's Lateral Geniculate Nucleus. Tohoku J. exp. Med., 1972, 106 (2), 209-210 — Relay neurons in the cat's lateral geniculate nucleus have either on-center or off-center receptive field. They are further classified into Type-I (phasic) and Type-II (tonic). Type-I cells are activated by faster conducting opfic tract fibers and send the visual information to visual cortex by faster conducting radiation fibers, while Type-II cells area ctivated by slower fibers and extend slower radiation fibers, while Type-II cells are activated by slower fibers and extend slower radiation fibers, while Type-II cells are activated by slower fibers and extend slower radiation fibers. - cat's lateral geniculate nucleus; receptive field; discharge pattern; conduction velocity

Most common type of receptive fields (RF) of cat's optic nerve fibers have been classified into Type-I (phasic) and Type-II (tonic) which contain on-center and offcenter subdivisions. The average conduction velocity of Type-I fibers is faster $(40 \mathrm{~m} / \mathrm{sec})$ than that of Type-II fibers (26 m/sec) (Fukada 1971, Saito et al. 1970).

To investigate the functional significance of the above two types of optic nerve fibers along the cat's visual pathway, single units were isolated in the cat's lateral geniculate nucleus (LGN) using tungusten microelectrodes. Cats were lightly anaesthetized with pentobarbital sodium and immobilized with gallamine triethiodide. A small spot of light $\left(1 / 10^{\circ}, 2.5 \times 10^{3} \mathrm{~cd} / \mathrm{m}^{2}\right)$ or a small black object $\left(1^{\circ}\right.$ circular spot) was displayed on the tangent screen $\left(17 \mathrm{~cd} / \mathrm{m}^{2}\right)$. For individual units, the response characteristics to the photic stimulation were examined. Simultaneously, the response latencies to single electric stimuli to the optic chiasm (OX), optic tract (OT) and visual cortex (VC) were measured. Recording sites were confirmed by making the electrolytic lesion by passing currents through the electrode tip.

More than three quarters of LGN units had concentric on-center or off-center RF. They were identified as the relay neurons which directly received the inputs from the OT fibers and extended their own axons to the VC; because when the latencies of the $\mathrm{OX}$ and OT stimulations were extrapolated to the zero distance (recording site), the time lags were around $1 \mathrm{msec}$. They were divided into two types: Type-I (phasic) or Type-II (tonic). Examples of discharge patterns of the on-center units are shown in Fig. 1. Records in the left column are responses to $50 \mathrm{msec}$ flash of small spot of light at the RF center, and those of the right column are initial parts of the responses to the long-lasting (more than 10 sec) stimulus. Off-center units were also divided into two types: Type-I responded only transiently when the small black object was presented at the center of the RF, while Type-II continued to respond. Thus, the relay neurons in LGB could be divided into Type-I and Type-II in the same way as the optic nerve fibers.

Received for publication, October 30, 1971. 


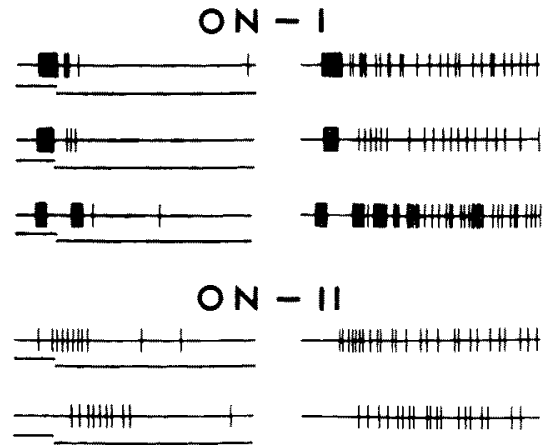

Fig. 1

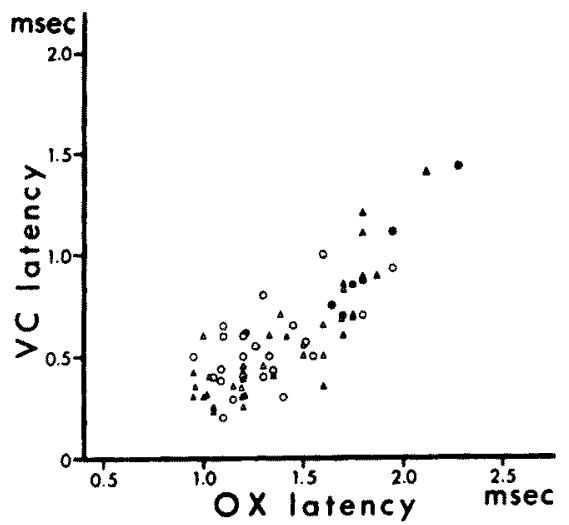

Fig. 2

Fig. 1. Discharge patterns of the on-center units. ON-I, on-center Type-I units; ON-II, on-center Type-II units. Type-I units respond with the burst of discharge. Further explanations see text.

Fig. 2. Correlation diagram for LGN relay cells between the latencies to OX stimulation and those to $\mathrm{VC}$ stimulation. Open circles represent on-center Type-I; open triangles, off-center Type-I ; filled circles, on-center Type-II ; and filled triangles, off-center Type-II.

Latencies to the $\mathrm{OX}$ stimulation were positively correlated with latencies to the VC stimulation (Fig. 2), and the correlation coefficient was 0.80 . A similar relation was found in the rat LGB by Noda and Iwama (1967). Latencies of Type-I units to the OX and VC stimulations were signifieantly shorter than respective latencies of Type-II units (average latency to $O X, 1.3 \mathrm{msec}$ versus $1.9 \mathrm{msec} ; \mathrm{VC}, 0.5 \mathrm{msec}$ versus $1.0 \mathrm{msec}$ ). It was concluded that the relay neurons which receive thicker OT fibers send thicker axons toward VC. Moreover, there exists a close relationship between the fiber diameter and the response characteristics to the visual stimuli.

\section{References}

1) Fukada, Y. (1971) Receptive field organization of cat optic nerve fibers with special reference to conduction velocity. Vision Res., 11, 209-226.

2) Noda, H. \& Iwama, K. (1967) Unitary analysis of retinogeniculate response time in rats. Vision Res., 7, 205-213.

3) Saito, H., Shimahara, T. \& Fukada, Y. (1970) Four types of responses to light and dark spot stimuli in the cat optic nerve. Tohoku J. exp. Med., 102, 127-133. 